« [Homère] distingue entre les noms données par les hommes et les noms donnés par les dieux. [...] Car bien évidemment, les dieux donnent, en accord avec la dénomination correcte, les noms qui sont précisément les noms par nature [...] il est permis d'opérer des variations avec les syllabes au point de faire croire à l'homme ordinaire que les noms diffèrent bien qu'ils soient identiques. [...] Comme nous le disions à l'instant Astuánax et Héktōr ne contiennent aucune lettre identique sauf le $\mathbf{t}$, et pourtant ils ont la même signification. » (Platon, Cratyle. Traduction de Catherine Dalimier. Paris : Flammarion, 1998, p. 88, 93) 


\title{
Epistémologie du nom propre en traduction
}

\author{
Michel BALLARD
}

Université d'Artois, Arras

France

\begin{abstract}
Résumé : Cette étude repose sur les principes de la traductologie réaliste, c'est-à-dire l'observation des produits de l'activité de traduction comme voie d'accès aux éléments et aux paramètres qui la constituent. La spécificité des noms propres constitue l'obstacle naturel à leur traduction au sens classique du terme mais leur potentiel sémantique peut, de façon naturelle ou par insertion dans des contextes de création littéraire ou pragmatique, générer des modes de traduction qui vont du littéralisme à la recréation, sans parler de ceux qui correspondent aux sollicitations de normes temporelles ou stylistiques. L'épistémologie du nom propre se construit en fin de compte dans le cadre d'une sémiotique qui intègre les fonctions du signe dans son contexte social de production et celles qu'on lui fait assumer dans les textes.
\end{abstract}

Mots-clés : observation, spécificité, sémiotique, sens, usage, contexte, fonctions, temps, texte, degrés d'appréciation et variations techniques.

Abstract: This study is based on the principles of realistic translatology, i.e., the observation of the artifacts of translation practice as a means of revealing the components and parameters of their production. The specificity of proper names constitutes the barrier to their translation in the classical sense of the term, but their semantic potentiality can, naturally or within the context of literary or pragmatic creations, generate modes of translation ranging from literalism to recreation, not to mention those related to time period and stylistic norms. In other words, the epistemology of proper names constitutes itself within the frame of a semiotic approach integrating the functions of the sign within its social context of production and the function it comes to bear within texts.

Keywords: observation, specificity, semiotics, meaning, usage, functions, time, text, degrees of appreciation and variational techniques.

Les déclarations des traductologues concernant des problèmes spécifiques sont aussi intéressantes pour elles-mêmes que pour ce qu'elles révèlent de leur rattachement à une épistémologie de la traductologie. La période de l'après-guerre mondiale a vu la linguistique réclamer la gestion de ce domaine ; puis, par réaction, les professionnels, au nom du savoirfaire, ont contesté cette capacité et généré des systèmes comme la Théorie interprétative. On trouve dans ces deux champs d'étude des déclarations 
concernant le nom propre ${ }^{1}$ en traduction qui témoignent à première vue d'une sorte de sens commun mais dont on ne peut percevoir toute la portée que si l'on creuse la surface de ces axiomes pour remonter à leur substrat épistémologique.

En 1955, Georges Mounin, dans Les Belles infidèles, déclare :

[...] la volonté d'atteindre à l'illusion d'un texte écrit directement dans notre langue [...] comporte tout au moins une limite infranchissable : les noms propres, qu'il faut garder dans la forme étrangère toutes les fois qu'elle n'est pas francisée. (Mounin [1955] 1994, 78) [nous soulignons]

Près de quarante ans après, dans son Manuel d’initiation à la traduction professionnelle de l'anglais vers le français, Jean Delisle expose un point de vue qui semble renvoyer au même principe de non-traduction :

Tout texte à traduire renferme une proportion variable d'éléments d'information qui échappent presque complètement à l'analyse du sens. Le traducteur les retranscrit tout simplement dans le TA sans vraiment avoir besoin d'interroger le contexte ou la situation pour en dégager le sens, d'où le terme « report » $[\ldots]$

Les éléments d'information faisant généralement l'objet d'un report sont les noms propres, les nombres, les dates, etc. (Delisle 1993, 124)

Et il est vrai que si l'on se réfère à la réalité, le nom d'un chef d'État traversera les ondes ou les salles de rédaction des journaux sous une forme écrite inchangée, avec toutefois sans doute de légères déformations à l'oral. Mais l'automobiliste qui roule en Belgique vers la France verra sur les panneaux une ville annoncée du nom de Reijsel et s'apercevra au bout de quelque temps qu'il s'agit de celle que l'on désigne en France du nom de Lille. Le touriste qui entre dans une librairie en Italie sera peut-être attiré par un livre sur la pinacothèque de Monaco, or, si Monaco peut effectivement correspondre à la capitale de la principauté de Monaco, il peut s'agir également de Münich, ville de Bavière. Genova en italien ne désigne pas la capitale de la Suisse, Genève, mais la ville que les Français désignent par Gênes. Et que dire de cette pièce de Shakespeare, The Merry Wives of Windsor, dont le traducteur Armand Robin a rendu les noms de personnages comme Robert Shallow et Abraham Slender par Abraham Létriqué et Robert Leborné ?

Comment se fait-il que les deux traductologues ci-dessus mentionnés ne tiennent pas compte de ces données? Nous avancerons l'hypothèse que la cause en est le substrat de leur théorisation.

\footnotetext{
${ }^{1}$ À partir de maintenant : Npr.
} 
2. The practice, didactics and critiques of translation / Pratique, didactique et critique de la traduction / Praxis und Didaktik des Übersetzens, Übersetzungskritik / Pratica, didattica e critica della traduzione / Práctica, didáctica y crítica de la traducción/

Georges Mounin, dans le passage évoqué, est en train de dessiner sa classification des manières de traduire (la célèbre opposition entre verres transparents et verres colorés), il est pris dans un mouvement d'élaboration global qui lui fait reléguer un problème comme le Npr au second plan, ou plutôt qui le rend aveugle à la spécificité ${ }^{2}$ de cet élément et le pousse à intégrer sa traduction dans le cadre d'une pratique qui serait fondée sur une opposition binaire (dont le fonctionnement n'est finalement pas assez précisé). En outre, et la suite de sa production (Les Problèmes théoriques de la traduction) le montrera largement, il est pris dans un mouvement de pensée fortement marqué par le structuralisme. Saussure déclarait :

Notre définition de la langue suppose que nous en écartons tout ce qui est étranger à son organisme, à son système, en un mot tout ce qu'on désigne par le terme de «linguistique externe ». Cette linguistique-là s'occupe pourtant de choses importantes, et c'est surtout à elles que l'on pense quand on aborde l'étude du langage.

Ce sont d'abord tous les points par lesquels la linguistique touche à l'ethnologie, toutes les relations qui peuvent exister entre l'histoire d'une langue et celle d'une race et d'une civilisation. [...]. (de Saussure 1976, 4043)

Déclarations auxquelles les propos suivants de Bakhtine semblent apporter une dénégation totale: "le signe et la situation sociale où il s’insère sont indissolublement liés. Le signe ne peut pas être séparé de la situation sociale sans voir s'altérer sa nature sémiotique » (Bakhtine 1977, 63, souligné par l'auteur).

La nature même de la traduction la place hors du champ d'une linguistique structurale dans la mesure où elle est faite d'un entrelacs de lecture, d'interprétation, de production d'équivalences et d'écriture qui demandent l'action d'un sujet gestionnaire d'un contact de langues et de cultures ayant pour objet un transfert dont la nature, en l'occurrence, demande à être précisée et sera sans doute éclairée par la prise en compte du paramètre culturel.

La position de Jean Delisle a davantage de quoi surprendre dans la mesure où il se situe dans la mouvance d'une théorisation qui est fondée sur l'interprétation et le sens. Peut-être faut-il faire intervenir ici le caractère trop généralisant de cette théorie qui se veut valable pour tous les types de textes, toutes les langues; c'est ce que l'on trouve exprimé en tête de l'ouvrage de Marianne Lederer sur La Traduction aujourd'hui :

\footnotetext{
${ }^{2}$ Le changement d'approche qui s'est opéré ces dernières années est visible dans les titres et les contenus des articles de Georgiana Lungu Badea (2011) et Thierry Grass (2011) présents dans la bibliographie.
} 
le processus de traduction est universel, indépendant de la paire de langues concernée ou de l'oeuvre de l'auteur. Quelles que soient les langues, quel que soit le texte, la démarche du traducteur est la même : la recherche du sens et sa réexpression sont le dénominateur commun à toutes les traductions. (Lederer 1994, 9) [nous soulignons]

Il convient également de faire intervenir la distance que cette théorie préconise avec la forme, l'accent mis sur la déverbalisation, le refus de la spécificité des genres et sans doute le refus du mot comme unité de traduction. Or, si l'on peut comprendre ce souci fort louable de ne pas être prisonnier des formes dans la reformulation, il est cependant indéniable qu'elles ont leur importance tant au niveau de la compréhension que de la réexpression.

Plutôt que du mot, il nous semble préférable de parler du signe à propos du nom propre et de l'étudier dans le cadre d'une sémiotique intégrée, comme le suggère la déclaration de Bakhtine à propos du signe en général ; parler du nom propre est une chose, parler de sa traduction en est une autre, qui suppose la référence à des corpus d'utilisation variés reflétant ses usages et ses traitements. La traductologie ne se décrète pas, elle se crée, elle s'élabore à partir de l'observation, en faisant intervenir des données d'autres sciences sans doute mais sans se laisser emporter par le courant ou dominer par les points de vue extérieurs.

Nous prendrons donc pour bases de notre approche du problème la dualité signifiant/signifié, le rattachement du signe à un système, son intégration dans un texte (lui-même réalisé dans le cadre d'un genre) ainsi que la question de sa fonction sociale, scripturale et esthétique. À cela nous ajouterons les caractéristiques de la traduction comme transfert linguisticoculturel visant à reproduire l'effet d'une production textuelle en tenant compte de son support et de la différence de public, sans oublier la prise en compte de la subjectivité du traducteur malgré une visée objective éthique idéale affichée.

La traduction commence par une lecture et à ce stade apparaît le fait que le nom propre est loin d'être un signe inerte, sa fonction primordiale de désignateur (et souvent de désignateur culturel) fait appel non seulement à une connaissance encyclopédique mais aussi au bon sens, au jugement, à l'attention. Encore une fois, les erreurs de traduction sont révélatrices à cet égard d'opérations effectuées de façon instinctive et rapide par le traducteur compétent.

Le Npr, souvent, a subi une opacification sémantique qui gomme son étymologie et avec elle sa fonction référentielle idéique ; il arrive que certains Npr (parfois de la volonté même de l'auteur) soient encore proches des noms communs ; voici un exemple de mauvaise lecture effectuée par un étudiant dans un contexte de traduction universitaire (donc de traduction d'un extrait sans contexte large) : 
2. The practice, didactics and critiques of translation / Pratique, didactique et critique de la traduction / Praxis und Didaktik des Übersetzens, Übersetzungskritik / Pratica, didattica e critica della traduzione / Práctica, didáctica y crítica de la traducción/

[Fin du monologue intérieur de Maskelyne]

The idea of anyone like Maskelyne developing intuition was delightful. Pursewarden gave a long crowing laugh and reached for his coat. (L. Durrell 1958, 144-145)

*L'idée que Maskelyne puisse développer son intuition était formidable. Le gardien du trésor partit d'un long rire retentissant et prit précipitamment son manteau. (Copie d'étudiant)

Proposition de traduction:

L’idée que quelqu'un comme Maskelyne puisse acquérir de l’intuition était savoureuse. Pursewarden émit un long gloussement de plaisir et saisit son manteau

Il s'agit là d'un étudiant travaillant en condition d'examen et n'ayant accès qu'à un extrait du roman, on pourrait donc à la rigueur l'excuser mais il n'empêche que son erreur révèle un mécanisme, qui dans l'exemple suivant se reproduit, cette fois chez un professionnel :

Large meals were prepared in this room, cauldrons of stew for the insatiate hunger of eight. Stews of all that grew on these rich banks, flavoured with sage, coloured with Oxo, and laced with a few bones of lamb. (L. Lee 1962, 16)

De copieux repas étaient préparés dans cette pièce, de pleines marmites de ragoût pour huit estomacs insatiables. Ragoûts de tout ce qui poussait sur ces rives fertiles, parfumés de sauge, colorés d'oxyde de carbone, et que garnissaient quelques os d'agneau. (P. Reumaux 1991, 21-22)

La traduction demande l'établissement d'un sens, et ici le traducteur n'a pas vu qu'il y avait une majuscule; il a fonctionné selon le principe de paronymie (mots proches par la forme et différents par le sens). Il n'a de toute façon pas identifié le nom propre et son sens : Oxo est un produit que l'on ajoute dans les préparations pour donner du goût. Cet élément pose d'ailleurs un problème d'équivalence culturelle car de toute évidence les français ne savent pas ce qu'est "Oxo », l'équivalent pour eux à l'époque était : « le bouillon Kub».

Le nom propre appartient à la langue par son signifiant, ses sonorités, son écriture, c'est ce qui constitue son iconicité. Une inscription en caractère cyrillique nous indique que nous avons affaire à du russe et même le procédé de translittération va véhiculer des sonorités qui signalent le rattachement à un type de langue: pour le russe, par exemple, les terminaisons en '-ov' : Lomonossov, Gorbatchov.

Le processus d'assimilation phonétique et graphique (de francisation pour les Français) va gommer, parfois à outrance, l'identité du nom propre et par là-même réduire sa capacité à remplir sa fonction de 
désignateur. Dans la mesure où le nom propre désigne un individu ou un lieu unique, il y a intérêt à ce que ces référents puissent être identifiés par tout lecteur, quelle que soit son origine. Celui qui a toujours entendu parler de "Florence », cherchera en vain ce nom sur une carte italienne, il ne trouvera que Firenze (là aussi les choses sont complexes car faut-il dire que Florence est une francisation de Firenze alors que la ville fut fondée en 59 avt J.-C. par des vétérans de César et reçu alors pour nom Florentia ?). C'est un domaine où le bon sens se heurte à l'usage, qui est d'assimiler, à tort, les noms propres. Un toponyme n'a pas, a priori, plus de raison d'être 'traduit' qu'un anthroponyme : il n'y a pas plus de raison de transformer München en Munich (ou Moskva en Moscou) que de transformer "Charles de Gaulle » en autre chose. L'usage aussi nous amène à parler de traduction alors qu'il faudrait nuancer ce qui se passe et le décrire en termes plus précis.

On se rend compte que se poser des questions de cet ordre amène à préciser ce que l'on entend par traduction : dire que le nom propre est intraduisible est inexact ; il n'a pas en principe, par sa nature, à être traduit si l'on entend par traduire "remplacer un élément d'une langue par un élément d'une autre langue, qui lui est équivalent », il constitue un invariant du texte d'origine. Lorsqu'un nom propre renvoie à un référent unique, sa forme en tant que désignateur devrait être préservée, il n'a pas à être traduit au sens d'être " transformé » ou remplacé par un autre terme ; mais, comme il est pris dans un mouvement de traduction au sens de transfert vers un texte équivalent à celui qui le contient à l'origine, il a besoin d'être transféré et de subir une opération de report, pour utiliser le terme proposé par Delisle. Il convient à ce stade d'effectuer une mise au point sur ce terme : Jean Delisle l'a introduit en 1993 dans son manuel, La Traduction raisonnée, pour désigner un des aspects du processus cognitif de la traduction, celui « consistant à transférer tout simplement du TD vers le TA des éléments d'information ( $\mathrm{Npr}$, nombres, dates, symboles, vocables monosémiques, etc.) qui ne nécessitent pas ou presque pas d'analyse interprétative » (Delisle 1993, 42). Nous avons repris ce terme dans notre étude sur le Npr (Ballard 2001) en marquant nos réserves quant à l'idée d'absence d'interprétation, mais nous lui avons donné une extension plus large ensuite dans nos études sur la traduction des référents culturels (cf. Ballard 2005, en particulier p. 131) en en faisant le désignateur d'un processus distinct de l'emprunt (phénomène collectif d'intégration d'un terme étranger dans une langue) alors que le terme de report s'avère utile pour décrire ce processus particulier de traduction qui n'intègre pas un terme dans la langue mais, de façon occasionnelle, dans un texte.

L'opération de traduction ne sera totale que lorsque le sens ou la valeur du nom propre auront été transférés par l'existence d'une connaissance partagée par le lecteur de traduction ou par le jeu d'un 
2. The practice, didactics and critiques of translation / Pratique, didactique et critique de la traduction / Praxis und Didaktik des Übersetzens, Übersetzungskritik / Pratica, didattica e critica della traduzione / Práctica, didáctica y crítica de la traducción/

transfert sémantique d'ordre textuel (l'incrémentialisation) ou paratextuel (la note). Tant que la référence du Npr est obscure, il ne fonctionne que par son iconicité et confère au texte traduit une couleur locale purement visuelle et sonore. Il est intéressant de voir que l'histoire de la traduction, souvent associée à l'histoire des échanges culturels, est allée vers une "révélation» des noms propres; la grécisation des Npr par Leconte de Lisle en est un exemple patent, et cette révélation n'est pas purement sonore, elle renvoie dans ce cas à la portée du nom : «Hercule » est le nom qui nous est venu du latin, il renvoie, dans l'esprit d'individus moyennement cultivés à un héros (et demi-dieu) dont la principale caractéristique est la force, à un point tel que ce nom est repassé dans la classe des noms communs pour désigner une catégorie d'homme; mais le retour à la forme grecque du nom, Herakles rend lisible, pour ceux qui en sont capables, au travers de son étymologie, le destin fatal du héros condamné à une série d'épreuves pour « la gloire d'Héra ».

Le nom propre possède une fonction fondamentale de désignateur social ou spatial mais il contient aussi du sens par sa motivation et l'histoire de l'individu qui le porte.

Pour ce qui est des anthroponymes, la motivation du Npr est liée à un acte de nomination variable dans ses procédures : l'antiquité judaïque, l'antiquité classique, la civilisation arabe offrent des procédures où l'acte de nomination renvoie à un événement, un trait lié à l'individu ; la création du surnom renvoie généralement à un trait lié à l'individu mais aussi parfois à une parole, à un incident; de façon plus classique et figée, l'acte de nomination courant s'effectue par héritage (pour le nom de famille) et par utilisation d'un donné (les prénoms en usage dans une communauté, qui souvent avant le $\mathrm{XX}^{\mathrm{e}}$ siècle renvoyaient à une religion, une mythologie perçue comme un héritage ou une protection); l'auteur de roman ou de pièces de théâtre peut utiliser toutes les ressources précédemment évoquées mais généralement le choix ne se fait pas au hasard: des considérations socioculturelles, la prise en compte de connotations interviennent généralement de façon plus ou moins forte.

La situation de traduction confronte le traducteur avec un texte constellé de Npr dont il doit identifier la nature et la fonction tout en tenant compte pour sa réexpression des données préexistantes en matière d'équivalences possibles. Les noms de famille, en principe, bénéficient du report mais leurs connotations sont parfois ainsi perdues et de toute façon le nom ne sera lu par le lecteur de traduction que pour son iconicité ; les noms de famille créés par un auteur posent le problème de leur transposabilité de façon plus ou moins aiguë : dans une bande dessinée telle que les Aventures d'Astérix, le traducteur se livre souvent à une véritable 
reconstitution du nom pour obtenir un effet analogue3. L'existence de prénoms équivalents dans la culture d'accueil ne facilite pas la tâche du traducteur car elle pose le problème de l'assimilation, et par voie de conséquence de la naturalisation du texte, le risque étant de créer un texte disparate où des personnages étrangers ont des noms français. Toutes sortes de considérations interviennent: un traducteur de théâtre comme Eric Kahane estime que parfois il est préférable d' « aménager » certains toponymes anglais : parce qu'ils peuvent se révéler difficiles à prononcer pour un acteur, parce qu'ils n'évoquent rien pour le spectateur français moyen; les aménagements prennent alors dans les cas dont il parle la forme de suppression ou de remplacement par un nom commun exprimant leur valeur générale ou textuelle. Parfois ce sera le sens et son utilisation dans le texte qui déclencheront la traduction ou l'utilisation de la note : dans la traduction roumaine de L'agonie de La Sémillante, « la traductrice considère nécessaire de traduire en bas de page le nom du navire, surtout parce que La sémillante qui signifie 'l'espiègle' $(1964,62)$ a une destinée si tragique, contrastant avec les qualités conférées par son appellation » (Rădulescu 2011, 326).

Outre les principes généraux déductibles de la structure et des fonctions du Npr (envisagés dans une stratégie de traduction), il convient de faire intervenir la localisation du Npr, c'est-à-dire son insertion dans un texte, dans un contexte. Une stratégie d'observation faisant intervenir la comparaison de l'original et de la traduction peut se décrire en termes de visibilité numérique. Un texte contient un certain nombre de noms propres, sa traduction peut afficher le même nombre, un nombre réduit, des effacements, des insertions.

Les causes des variations en densité sont de plusieurs natures: historiques, sociologiques, linguistiques, stylistiques. J'en examinerai successivement deux types : celles qui sont liées à une forme de prévenance de la part du traducteur vis-à-vis de son public, et puis celles qui relèvent davantage de sortes de normes d'écriture.

Le premier type de variation fait bien entendu penser d'abord à la technique des belles infidèles; mais même dans un cadre général de préservation de l'intégrité du texte original, les raisons avancées par certains traducteurs soucieux d'efficacité et d'assurer un certain confort de lecture, ne manquent pas de poids. Au XVII e siècle, 1646, Perrot d'Ablancourt, traduisant Les Guerres d'Alexandre d'Arrien, estime (comme il l'estimera plus tard à propos des proverbes et comparaisons de Lucien en

\footnotetext{
3 Nous renvoyons sur ce point à l'analyse très fouillée qu'en a fait Catherine Delesse dans le chapitre 1 de Delesse \& Richet (2009).
} 
2. The practice, didactics and critiques of translation / Pratique, didactique et critique de la traduction / Praxis und Didaktik des Übersetzens, Übersetzungskritik / Pratica, didattica e critica della traduzione / Práctica, didáctica y crítica de la traducción/

16544) que toutes ces références sont oubliées et risquent d'ennuyer le lecteur 'moderne' :

Je me contenteray de dire qu'il y a une si grande quantité de noms propres dans cette Histoire, que j'ay esté contraint d'en rejetter une partie dans les marges ou dans les remarques. Car il est certain qu'il n'y a que les principaux de necessaires dans une narration, le reste ne sert qu'à l'embrouiller, ou à faire oublier les autres ; Outre que les Grecs ont accoustumé de mettre le nom du pere avec celuy du fils, et quelquefois celuy de la charge ou de la nation ; de sorte qu'il s'en trouve icy jusqu'à quinze ou vingt de suite, ce qui cause non seulement du degoust, mais de l'obscurité, principalement dans le recit des batailles où l'on ne sçauroit estre trop clair. (D’Ablancourt [1646] 1972, 140)

D’Ablancourt défend ici un aménagement du texte en fonction de l'horizon d'attente d'un public qui risque de ne pas comprendre ou d'être rebuté par une manière de dire qui s'encombre de références onomastiques que l'on n'utilise plus.

Au siècle suivant, le Joseph Andrews de Fielding, traduit par l'abbé Desfontaines en 1743, subit des variations en densité onomastique de deux ordres.

Les premières concernent des suppressions de Npr que Desfontaines, à la manière de D'Ablancourt ci-dessus, estime obscurs ou sans intérêt pour le lecteur français, c'est ce qui ressort des notes ajoutées par l'éditeur hollandais de la version de 1744:

* Le Traducteur a négligé de traduire ce qui commence par ces mots, So I have seen etc. jusqu'à la fin du Chapitre; supposant avec raison que cela auroit aucun sel en François, à cause qu'il ne s'y agit que d'une comparaison de personnes uniquement connues à Londres. Le but de cette comparaison est visiblement de se moquer de certains Auteurs Anglois, qui se plaîsent à faire des comparaisons sans goût \& sans jugement. (Note de l'édition de 1744 citée dans K. Taivalkoski 2006, 98)

En réalité, ce passage n'a rien d'intraduisible, il contient des noms de personnages renvoyant à des types (sur le mode des moralités) :

[...] and thus the poor Lady was tortured with Perplexity; opposite Passions distracting and tearing her Mind different ways.

So I have seen, in the Hall of Westminster; where Serjeant Bramble hath been retained on the right Side, and Serjeant Puzzle on the left, the Balance

4 «L'auteur allègue à tout propos des vers d'Homère, qui seraient maintenant des pédanteries, sans parler de vieilles fables trop rebattues, de proverbes, d'exemples et de comparaisons surannées, qui feraient à présent un effet tout contraire à son dessein [...]. » (D’Ablancourt 1988 [1654] : 23) 
of Opinion (so equal were their Fees) alternately incline to either Scale. (Fielding 1961, 30)

Le passage précédent contient un développement plus général où Fielding fait intervenir des personnages allégoriques comme Love, Pity, Revenge; tout cela a été dûment traduit dans la traduction française de Suzanne Nétillard et Paul Vigroux, publiée chez les Éditeurs français réunis en 1955 .

Les autres variations concernent des étoffements qui tirent le texte vers le public français et satisfont aussi l'ego du traducteur; il s'agit de références à Desfontaines lui-même et à Voltaire à propos des considérations littéraires dont Fielding agrémente son récit. C'est ainsi que lorsque dans la préface, Fielding parle du Télémaque de Fénelon, Desfontaines, sous couvert du pseudonyme qu'il a adopté pour cette traduction (La Dame anglaise), ajoute en note :

* M. l'Abbé Desfontaines est d'un sentiment différent dans quelque endroit de ses Observations, que je ne me rappelle pas, \& M de Voltaire pense comme lui, dans son Essay sur la Poësie Epique. Mais je crois que l'un \& l'autre se trompent [!]. Le Telemaque est un vrai Poëme [...].» (Desfontaines 1990, 38)

« Rousseau » est introduit dans le chapitre $17 \mathrm{du}$ livre I, et une autre référence à Voltaire est insérée plus loin dans le roman lorsque Fielding disserte sur les normes de divisions des oeuvres en chapitres ou en chants :

Ce n'est sans doute que pour se conformer à l'usage établi, que Voltaire, sur les pas des vrais Poëtes épiques, a partagé en dix beaux Chants son historique Henriade. » (Desfontaines 1990, 129)

Le second type de variation relève de manières d'écrire, que certains qualifient de normes; certaines configurations textuelles peuvent également générer des différences de densité notoires entre les textes traduits et leurs originaux.

Dans une étude de la traduction du roman d'Orwell, 1984, nous avons noté, sur 15 pages de texte anglais, la présence de 32 mentions du nom du personnage principal contre 42 mentions dans la version française. La cause principale de ces ajouts ou dépronominalisations est le souci de clarification du texte ; par exemple voici une dépronominalisation, motivée par le désir de lever une ambiguïté par rapport à des pronoms se référant à un objet, « le télécran », thème de la phrase précédente :

The instrument (the telescreen, it was called) could be dimmed, but there was no way of shutting it off completely. He moved over to the window. (Orwell 1961: 5) 
2. The practice, didactics and critiques of translation / Pratique, didactique et critique de la traduction / Praxis und Didaktik des Übersetzens, Übersetzungskritik / Pratica, didattica e critica della traduzione / Práctica, didáctica y crítica de la traducción/

Le son de l'appareil (du télécran comme on disait) pouvait être assourdi, mais il n'y avait aucun moyen de l'éteindre complètement. Winston se dirigea vers la fenêtre. (Audiberti 1972, 12)

Une étude analogue menée sur les premières pages du roman d'Iris Murdoch, The Bell, et sa traduction par Jérôme Desseine, révèle une pratique très différente. Le texte anglais offre une très forte densité : 51 occurrences du nom de l'héroïne, « Dora », dans les pages 7 à 15, contre 10 mentions dans la traduction. Le principe de réduction dans ce cas est déclenché par le principe esthétique de non-répétition; outre la pronominalisation, on a l'utilisation par le traducteur de périphrases descriptives utilisant des aspects de l'héroïne :

Dora had accepted his proposal of marriage. (Murdoch 1963, 7)

[...] La jeune fille accepta sa demande en mariage. (Desseine 1985, 10)

When one man, a violonist, had taken a more personal interest in Dora [...]. (Ibid., 9)

[...] lorsqu'un jour un violoniste sembla s'intéresser à sa personne [...]. (Ibid., 12)

Before their marriage Paul had warned Dora that they were likely to quarrel. (Ibid., 9)

Avant leur union, il avait averti sa fiancée qu'ils se querelleraient vraisemblablement. (Ibid., 12)

Cette rhétorique de la périphrase est couramment pratiquée dans les textes journalistiques et même sans que le risque de répétition soit impliqué. Un journaliste ou un présentateur de télévision se sentirait déshonoré de parler de Marseille, il utilisera régulièrement «la cité phocéenne », de même la personne présentant la météorologie parlera toujours de l' "hexagone" et non de la France; pour cette raison la traduction d'articles de la presse française en anglais donnera lieu à un accroissement de Npr dans la mesure où cette rhétorique de la périphrase semble moins pratiquée 5 dans la « langue de Shakespeare ».

En fin de compte, le grand générateur de traduction, au sens classique du terme, est le sens : on traduira plus volontiers des surnoms ou des noms symboliques que des noms classiques ayant simple fonction de désignateurs. Voici un exemple de signe dont on peut observer les degrés de traduction selon la sollicitation par le contexte, il s'agit de Home counties.

\footnotetext{
5 Mais, comme chacun sait, il y a toujours des 'exceptions' pour confirmer la règle ou en l'occurrence, la tendance: dans sa préface Fielding dit: "Thus the Telemachus of the archbishop of Cambray appears to me of the epic kind » (1961, XVIII), ce que Desfontaines traduit par: "Le Telemaque de Monsieur de Fénelon me paraoît autant mériter le titre de Poème épique » $(1990,38)$.
} 
Au début du roman de Golding, Lord of the Flies, Ralph, l'un des enfants rescapés de l'accident d'avion, sort de la jungle et se dirige vers la lagune :

The fair boy stopped and jerked his stockings with an automatic gesture that made the jungle seem for a moment like the Home Counties. (W. Golding, 7)

Deux dangers guettent le traducteur avec un terme de ce genre dans ce contexte. Le premier consisterait à utiliser la traduction-définition du dictionnaire: « les comtés qui entourent Londres » (Robert \& Collins) « les comtés avoisinant Londres » (Harrap's) « comtés limitrophes de Londres » (Hachette Oxford), ce qui donnerait une formulation assez gauche dans ce contexte. Le second risque est constitué par le saut culturel que représente l'adaptation ou naturalisation ne tenant compte que de la culture du public d'arrivée, et qui rendrait cet élément par quelque chose du style: «l'Ile de France ». La traductrice, Lola Tranec, a trouvé le terme qui à la fois explicite le référent et en suggère les connotations, tout en préservant la référence à son anglicité :

Le garçon blond s'arrêta et [remonta] ses chaussettes d'un geste machinal. L'espace d'une seconde, son geste évoqua le coeur de l'Angleterre et la jungle fut oubliée. (L. Tranec, 9)

On perçoit là toute la différence entre une traduction créative et une «traduction» de dictionnaire, qui nous fournit des équivalences préétablies valables pour une utilisation du terme en fonction primaire, comme simple désignateur. Par exemple si l'on traduit un passage où quelqu'un dit : "I've just bought a book on the Home Counties : je viens d'acheter un livre sur les comtés qui entourent Londres ».

Si l'on consulte les dictionnaires unilingues, The Home Counties n'apparaît pas dans le Longman Dictonary of Contemporary English, par contre dans le Longman Dictionary of English Language \& Culture, on a une bonne description du terme et de son potentiel :

The area of SE England around London, including the counties of Hertfordshire, Essex, Kent, Surrey, East and West Sussex, Berkshire and Buckinghamshire. People who live in the Home Counties are typically thought of as being middle class and conservative and as having a comfortable life. (LDELC)

Comme on le voit, la définition est d'abord informative et géographique, elle correspond à la traduction avec un degré zéro de connotation, l'utilisation qu'en fait Golding est déjà plus sentimentale et l'exemple suivant montre une utilisation du potentiel de la description 
2. The practice, didactics and critiques of translation / Pratique, didactique et critique de la traduction / Praxis und Didaktik des Übersetzens, Übersetzungskritik / Pratica, didattica e critica della traduzione / Práctica, didáctica y crítica de la traducción/

sociologique de la région fournie dans la seconde phrase de l'article du dictionnaire :

She was very nearly a pretty girl in a typically unambitious English Home Counties sort of way, but she was prevented from achieving this modest beauty by her nose.It was long and thin and turned up sharply at the end like a ski-jump. (Boyd 1981, 33)

C'était presque une de ces jolies filles bon chic bon genre du style province, mais quelque chose lui interdisait ce modèle standard : son nez. Il était long, mince et relevé comme un tremplin de ski. (Besse 1984, 37)

Dans le premier exemple, c'est plutôt le côté sentimental du terme qui est traduit, dans le second c'est son aspect sociologique : un lieu proche de la capitale mais qui n'est plus la capitale.

\section{Conclusion(s)}

Le nom propre a pour fonction première (sociale) d'être un désignateur de personne, d'être, de lieu, d'institution; cette fonction première d'identification et de repérage d'un référent, en principe unique, lui confère en traduction une sorte d'immunité qui induit sa préservation et oriente le traducteur vers une stratégie de report qui assure une présence étrangère dans un texte, que la traduction (vue dans son sens large) a baptisé " couleur locale », celle-ci pouvant d'ailleurs exister dans un texte non traduit par simple désir ou volonté de marquer une présence : un texte français peut contenir des régionalismes, perçus comme exotiques par rapport à la langue standard. L'usage qui consiste à aménager le $\mathrm{Npr}$ est révélateur d'une propension naturelle à naturaliser l'étranger et de la difficulté à accepter l'autre, la différence.

Par sa nature de signe, et son histoire, le nom propre est signifiant et cette signifiance peut être sollicitée ou ranimée par l'auteur ou/et le contexte. La signifiance agit alors comme force obligeant le traducteur à pratiquer une opération de transfert plus ou moins équilibrée : l'incrémentialisation est un processus qui permet de ménager la conservation du signe étranger et d'y accoler sa valeur; la sémantisation pure et simple de la valeur du Npr peut apparaître comme une perte d'iconicité aux partisans de la préservation de l'étrangeté du $\mathrm{Npr}$, elle ne l'est pas vraiment dans la mesure où grâce à la créativité du traducteur elle préserve l'objet de l'utilisation du Npr dans l'original, sa fonction. 


\section{Références bibliographiques}

Ablancourt, Nicolas Perrot d'. «Epître dédicatoire ». In : Lucien. Histoire véritable. Traduction et présentation par Perrot d'Ablancourt. Arles : Actes Sud, 1988 [1654] : 17-27.

Ablancourt, Nicolas Perrot d'. Lettres et préfaces critiques. Introduction, notices, notes et lexique par Roger Zuber. Paris : Didier, 1972.

Ballard, Michel. Le Nom propre en traduction. Paris : Ophrys, 2001.

Ballard, Michel. Numele proprii în traducere. Traduction roumaine réalisée sous la direction de Georgiana Lungu-Badea. Timişoara: Editura Universității de Vest, 2011.

Ballard, Michel. «Les stratégies de traduction des désignateurs de référents culturels ». In : Michel Ballard (éd.). La Traduction, contact de langues et de cultures (1). Travaux du CERTA. Arras : Artois Presses Université, 2005 : 125-151.

Bakhtine, Mikhail, Volochinov, V.N. Le Marxisme et la philosophie du langage. Essai d'application de la méthode sociologique en linguistique. Paris : Éditions de Minuit, 1977 [1929].

Delesse, Catherine, Richet, Bertrand. Le Coq gaulois à l'heure anglaise. Analyse de la traduction anglaise d'Astérix. Arras : Artois Presses Université, 2009.

Delisle, Jean. La Traduction raisonnée. Manuel d'initiation à la traduction professionnelle de l'anglais vers le français. Ottawa: Presses de l'Université d'Ottawa, 1993.

Grass, Thierry, «Médecins sans frontières, Doctors without borders, Ärzte ohne Grenzen : traduire les noms propres d'associations (français-anglais-allemand) ». In: Tatiana Milliaressi (éd.). De la linguistique à la traductologie (Interpréter/traduire). Lille : Presses universitaires du Septentrion 2011 : 253-270. Lederer, Marianne. La traduction aujourd'hui. Le modèle interprétatif. Paris : Hachette, 1994.

Lungu-Badea, Georgiana, "Un panorama de la traduction roumaine des noms propres ». In : Tatiana Milliaressi (éd.). De la linguistique à la traductologie (Interpréter/traduire). Lille : Presses universitaires du Septentrion 2011 : 161-177. Mounin, Georges. Les Belles Infidèles. Lille : P.U.L., 1994 [1955].

Mounin, Georges. Les Problèmes théoriques de la traduction, Paris, Gallimard, 1963 .

Rădulescu, Anda. "Escamotage des tabous dans la traduction en roumain des récits français pour enfants au nom d'un idéal communiste ». In : Michel Ballard (éd). Censure et traduction. Arras : Artois Presses Université, $2011: 317-328$.

Saussure, Ferdinand de. Cours de linguistique générale. Édition critique préparée par Tullio de Mauro. Paris : Payot, 1976 [1915].

Taivalkoski-Shilov, Kristiina. La Tierce Main. Le discours rapporté dans les traductions françaises de Fielding au XVIII ${ }^{e}$ siècle. Arras: Artois Presses Université, 2006.

\section{Corpus}

Boyd, William. A Good Man in Africa. Harmondsworth : Penguin, 1982 [1981]. 
2. The practice, didactics and critiques of translation / Pratique, didactique et critique de la traduction / Praxis und Didaktik des Übersetzens, Übersetzungskritik / Pratica, didattica e critica della traduzione / Práctica, didáctica y crítica de la traducción/

Boyd, William. Un Anglais sous les tropiques. Traduction de Christiane Besse. Paris : Seuil, 1986 [1984].

Fielding, Henry. Joseph Andrews. New York : Holt, Rinehart, 1961 [1742].

Fielding, Henry. Joseph Andrews. Traduction de l'abbé Desfontaines. Édition de Serge Soupel. Paris : Garnier-Flammarion, 1990.

Fielding, Henry. Les Aventures de Joseph Andrews. Traduction de Suzanne Nétillard et Paul Vigroux. Préface et appendice de Pierre Daix. Paris : Éditeurs français réunis, 1955 .

Durrell, Lawrence. Mountolive. London : Faber \& Faber, 1958.

golding, William. Lord of the flies. Harmondsworth : Penguin, 1965 [1954].

Golding, William. Sa Majesté des mouches. Traduction de Lola Tranec. Paris : Gallimard, 1956.

Murdoch, Iris. The Bell. Harmondsworth : Penguin, 1963 [1958].

Murdoch, Iris. Les cloches. Traduction de Jérome Desseine. Paris : Gallimard, 1985.

Orwell, George. Nineteen Eighty-four. Harmondsworth : Penguin, 1961 [1949].

Orwell, George. 1984. Traduction d'Amélie Audiberti. Paris : Gallimard, 1972 [1950].

Lee, Laurie. Cider with Rosie. Harmondsworth : Penguin, 1962 [1959].

Lee, Laurie. Rosie ou le goût du cidre. Traduction de Patrick Reumaux. Paris : Phébus, 1991.

Shakespeare, William. The Merry Wives of Windsor / Les Gaillardes épouses de Windsor. Traduction d'Armand Robin. Édition des Euvres Complètes de Shakespeare. Paris : Club français du Livre, 1963, vol. 7. 